\title{
Importance ethnobotanique de Leptadenia pyrotechnica (Forssk.) Decne. dans le département de Diffa au Niger
}

\author{
Abdoul Aziz ELHADJI SANOUSSI ISSOUFOU ${ }^{1 *}$, Habou RABIOU ${ }^{1}$, \\ Idrissa SOUMANA ${ }^{2}$, Maman Kamal ABDOU HABOU ${ }^{1}$ et Ali MAHAMANE ${ }^{1-3}$ \\ ${ }^{1}$ Faculté des Sciences Agronomiques, Université de Diffa, BP 78, Diffa, Niger. \\ ${ }^{2}$ Institut National de la Recherche Agronomique du Niger; BP: 429: Niamey, Niger. \\ ${ }^{3}$ Faculté des Sciences et Techniques, Université Abdou Moumouni de Niamey, BP : 10662, Niamey, Niger. \\ *Auteur correspondant ; E-mail : sanoussiabdoulaziz@yahoo.fr; Tel: (+227)96344430.
}

\section{RESUME}

En zone sahélienne où les ressources ligneuses sont relativement rares, l'espèce Leptadenia pyrotechnica joue un rôle très important dans la vie des populations locales. La présente étude dont l'objectif est de répertorier les connaissances locales sur les modes d'utilisation et de gestion des peuplements naturels par les populations locale de Leptadenia pyrotechnica a été conduite dans la région de Diffa. Des enquêtes ethnobotaniques ont été conduites au niveau de cinq villages. Il s'agit de: Issari; Guelborno Mounimari, Jalori, Joulou Kriyé et le marché de Digurdi. Dans l'ensemble des villages, un total de 150 personnes a été interviewé à travers des entretiens individuels. Les principales rubriques du questionnaire administré sont relatives aux principales utilisations et leur impact sur la ressource. Il ressort de l'étude que toutes les parties de Leptadenia pyrotechnica (feuilles; rameaux, tiges, racines, écorce et la sève) sont utilisées par les différents groupes ethniques et socioprofessionnels de la zone d'étude (Haoussa, Peulh et Kanouri). Cependant, la connaissance de l'importance et les différentes catégories d'utilisation dépendent d'un groupe à un autre. Les principales catégories d'utilisations dénombrées dans le cadre de la présente étude sont: l'utilisation comme bois énergie, fixation des dunes, fourrage et pharmacopée traditionnelle. La fixation des dunes et la transhumance s'avèrent être les principaux facteurs limitant la disponibilité de cette espèce. Pour une conservation durable de l'espèce, une norme de prélèvement de Leptadenia pyrotechnica doit être définie pour la fixation des dunes et la capacité de charge des formations à Leptadenia pyrotechnica doit être contrôlée vis-à-vis des transhumances.

(C) 2020 International Formulae Group. All rights reserved.

Mots clés: Leptadenia pyrotechnica, utilisation, bois, sève, rameaux, racine.

\section{Ethnobotanical importance of Leptadenia pyrotechnica Forsk. in the department of Diffa in Niger}

\begin{abstract}
In the Sahelian zone where wood resources are relatively rare, the species Leptadenia pyrotechnica plays a very important role in the lives of local populations. The purpose of this study, which is to identify local knowledge on the use and management of natural stands by local populations of Leptadenia pyrotechnica, was conducted in Diffa region. Ethnobotanical surveys were conducted at five villages. These are: Issari; Guelborno
\end{abstract}


Mounimari, Jalori, Joulou Kriyé and Digurdi market. In all the villages, a total of 150 people were interviewed through individual interviews. The main sections of the questionnaire administered relate to the main uses and their impact on the resource. The study shows that all parts of Leptadenia pyrotechnica (leaves, twigs, stems, roots, bark and sap) are used by the different ethnic and socio-occupational groups in the study area (Hausa, Fulani and Kanuri). However, knowledge of importance and the different categories of use depend on one group to another. The main categories of uses enumerated in this study are: wood energy use, dune fixation, fodder and traditional pharmacopoeia. The fixation of the dunes and the transhumance appear to be the main factors limiting the availability of this species. For a sustainable conservation of the species, a Leptadenia pyrotechnica sampling standard must be defined for the fixation of the dunes and the carrying capacity of Leptadenia pyrotechnica formations must be checked against transhumance.

(C) 2020 International Formulae Group. All rights reserved.

Keywords: Leptadenia pyrotechnica, use, wood, sap, twigs, root.

\section{INTRODUCTION}

A l'instar des autres pays du sahel, le Niger est un pays dont le développement socioéconomique repose sur l'exploitation des ressources naturelles (Boubacar, 2010). Les produits divers fournis par les ligneux présentent des enjeux socio-économiques certains, car la vie de nombreuses populations en dépend directement (Bellefontaine et al., 2001 ; Abdou et al., 2019). Cependant, les changements climatiques et les actions anthropiques telles que la déforestation, la surexploitation des ressources naturelles, l'agriculture, le surpâturage et les feux de brousse contribuent actuellement à la perte de multiples plantes indigènes à importance capitale (Assogbadjo et al., 2011). Au Sahel en général et au Niger en particulier, les espèces ligneuses jouent un rôle important dans les ménages de la population rurale. L'utilisation des différentes espèces ligneuses dans une zone donnée se fait généralement en fonction de leur abondance. Cependant, il existe des espèces dont l'importance s'avère particulière pour certaines communautés. Les espèces qui se trouvent dans cette situation sont convoitées et exploitées sans tenir compte de leur abondance ni de leur taux de régénération, ce qui les menace de disparition (Abdou et al., 2014).

Une bonne partie de la zone sahélienne du Niger est déclarée zone pastorale (FSRGTT, 2010). L'augmentation du cheptel dans cette zone a engendré une forte pression pastorale avec comme conséquence, la remobilisation des sols rendant ainsi le sable mouvant (PADL DIFFA, 2010). Actuellement, le désert avance de $120000 \mathrm{Km}$ chaque année (Planetoscope, 2019). Pour pallier ce problème d'avancée du désert, la seule alternative est la fixation mécanique des dunes avec du matériel végétal. Le matériel utilisé au Niger est composé essentiellement des tiges de L. pyrotechnica. L'exploitation des tiges de cette dernière se fait sans aucun respect de normes et cela se traduit par la dégradation de ces peuplements. Plusieurs auteurs (Gulshan et al., 2012; Schmelzer Gurib-Fakim, 2013; Niaz et al., 2013; Verma et al., 2014 ; Fahim et al., 2015) ont souligné l'importance de L. pyrotechnica dans la vie socioéconomique de la population. $L$. pyrotechnica est traditionnellement utilisé à des fins différentes. Au Pakistan, une décoction de tige est prise comme antihistaminique et un expectorant tandis qu'en Inde, il est utilisé pour traiter la goutte et les rhumatismes. Au Yémen les tiges écrasées sont appliquées sur les plaies pour arrêter le saignement. Une infusion des parties aériennes est prise comme un diurétique pour traiter les troubles rénaux. Les Brindilles sont macérées et le liquide est pris pour traiter la rétention urinaire. L'infusion de la plante entière mélangée avec du lait de beurre est donnée pour traiter le prolapsus, l'estomac et les troubles utérins. Toutes les parties de $L$. pyrotechnica sont utilisées dans les remèdes populaires pour guérir la peau, le Musculo / squelette et les troubles gynécologiques. La fumée de la plante brûlée est utilisée pour traiter les maux de tête au Pakistan et les 
rhumatismes au Soudan. La sève de la plante est appliquée sur la peau pour traiter la variole, le psoriasis, l'eczéma et la dermatite (Gulshan et al., 2012; Schmelzer GuribFakim, 2013; Niaz et al., 2013; Verma et al., 2014 ; Fahim et al., 2015). Le jus des feuilles est traditionnellement utilisé pour le traitement de l'asthme, les rhumatismes et la diarrhée infantile. Pour enlever l'épine, la pâte de feuille est préparée et appliquée sur la blessure d'épines (Gulshan et al., 2012 ; Verma et al., 2014, Fahim et al., 2015).

Les formations à $L$. pyrotechnica de la région de Diffa sont menacées par l'ensablement, la déforestation et l'effet de broutage. La connaissance des différents usages liés à cette espèce permettra de mieux comprendre les menaces pesant sur celle-ci dans cette région. L'objectif de cette étude est d'évaluer les indicateurs d'utilisation et répertorier les connaissances endogènes sur $L$. pyrotechnica. Les informations collectées permettront d'apporter une contribution dans l'élaboration d'un plan de gestion efficace dans la zone où les ressources ligneuses sont relativement rares.

\section{MATÉRIEL ET MÉTHODES Zone d'étude}

La zone d'étude est située à l'extrême Est du Niger au nord de la Région de Diffa dans le Département de Diffa, commune rurale de chetimarie (Figure 1). La population de la zone est composée essentiellement de Kanouri, de Haoussa et de Peulhs. Les activités socioéconomiques de la zone d'étude sont dominées par l'élevage, l'agriculture et le commerce. Le Climat est tropical sec de type sahélien avec une pluviométrie moyenne annuelle de $400 \mathrm{~mm}$, entre 1950 et 2000 . Les sols de la zone d'étude sont dunaires. Dans cette zone a vocation pastorale, le déficit fourrager de la longue saison sèche entraine le déplacement des éleveurs à la recherche du pâturage. La végétation est dominée par des steppes à Balanites aegyptiaca, Acacia tortilis, Prosopis juliflora, L. pyrotechnica. Le choix de cette zone est basé sur la densité et les menaces pesant sur cette espèce au Niger.

\section{Échantillonnage}

Quatre villages ont fait l'objet d'investigation : Issari, Mounimari, Jalori et Joulou kriyé Ainsi que le marché de Digurdi. Cent cinquante personnes ont été enquêtées dont cent vingt parmi la population locale, quinze artisans et vendeurs du bois et quinze thérapeutes, soit, un échantillon de $15 \%$ de la population du terroir.

\section{Collecte des données}

La collecte des informations a été effectuée par une enquête formelle à l'aide des fiches destinées à la population locale, des fiches destinées aux artisans et vendeurs des bois et des fiches destinées aux thérapeutes. Les questionnaires ont été destinés aux répondants et à des personnes ressources afin de recueillir des informations d'ordre général et spécifiques sur les relations possibles entre les hommes et l'espèce $L$. pyrotechnica (Rabiou et al., 2017). À travers les différents questionnaires, les données suivantes ont été recueillies : a) les usages et les usagers de l'espèce ; b) la survie de l'espèce face aux différentes pratiques anthropiques ; c) les impacts socioculturels et écologiques sur l'espèce. Les questionnaires utilisés auprès des répondants étaient regroupés autour des thèmes suivants: (i) : Profil des répondants ; l'importance de cette section était de créer un climat de confiance entre les paysans et les enquêteurs. (ii) : Information et connaissance sur l'espèce L. pyrotechnica ; cette partie a permis au préalable de préparer les paysans à l'interview, d'introduire l'objet de cette étude sur les connaissances et l'importance de $L$. pyrotechnica, ainsi que le nom local de l'espèce. (iii) : Usages des organes de $L$. pyrotechnica par les groupes socioculturels. Ceci avait pour but de recenser l'ensemble des usages liés à tous les organes de $L$. pyrotecnica. (iv) : Traitement des maladies à partir des organes de L. pyrotechnica. Cette section a permis de répertorier les maladies traitées avec les organes de $L$.pyroetchnica ainsi que l'influence de la collecte de ces organes sur la dynamique des peuplements naturels. (vi) : Identification des traits socioculturels des usagers de la zone d'étude ; 
a permis d'appréhender les perceptions paysannes sur les groupes sociolinguistiques et leur implication dans l'usage des produits de L. pyrotechnica.

\section{Traitement des données}

Les données collectées lors des enquêtes ont été saisies à l'aide du tableur
Excel. Les calculs des fréquences de citation et l'élaboration des graphiques ont été effectués toujours au moyen du tableur Excel. Les types d'utilisation des bois et les villages d'enquête ont été soumis à une Analyse en Composantes Principales (ACP) à l'aide du Logiciel Minitab 14.

\section{Localisation commune rurale de Chetimari}
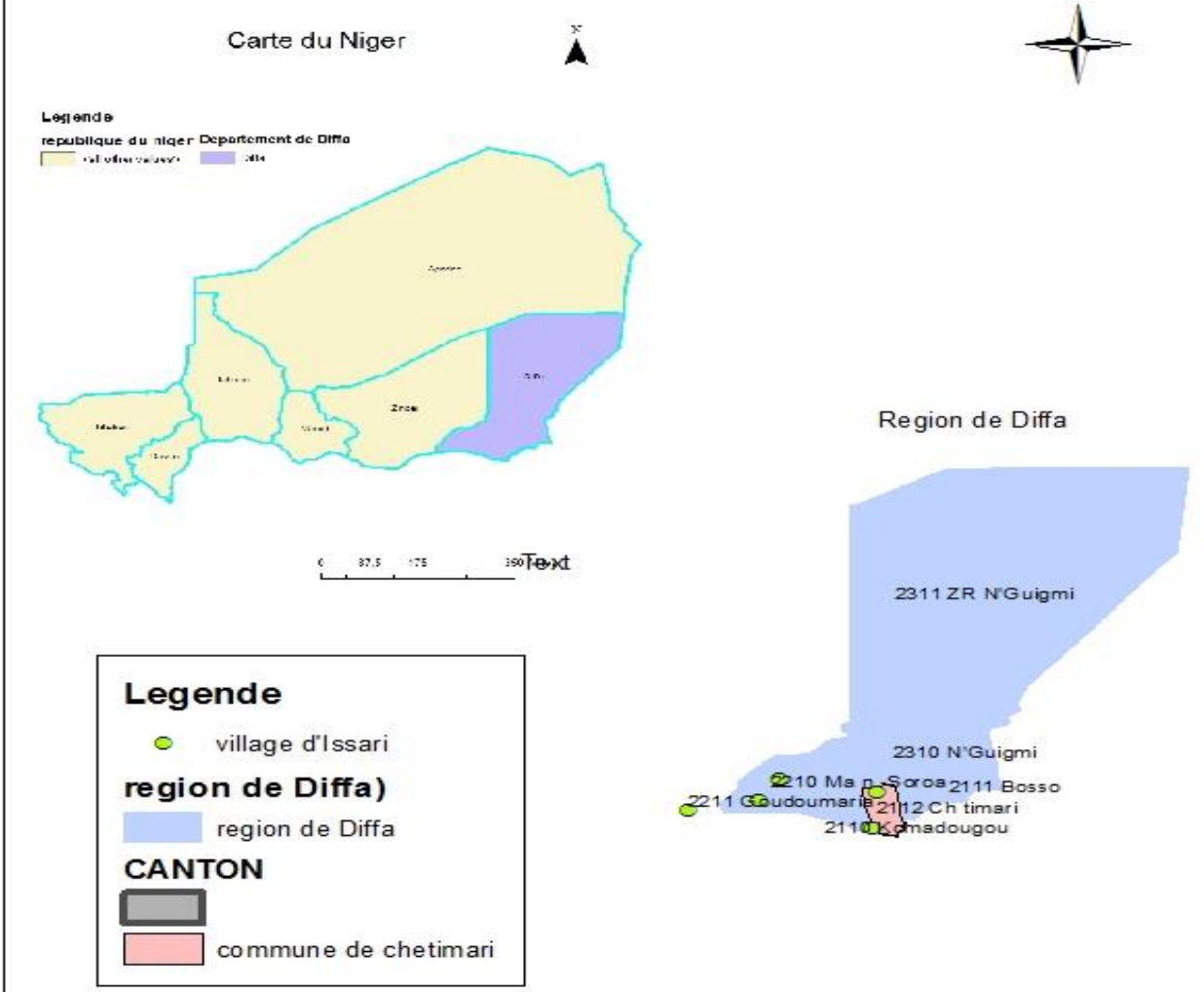

Figure 1 : Localisation de la zone d'étude. 


\section{RÉSULTATS}

\section{Connaissances pyrotechnica \\ endogènes de $L$.}

La plupart des personnes enquêtées utilise les organes de L. pyrotechnica excepté dans le village de Guelborno où le pourcentage de non utilisateurs est plus élevé. Les pourcentages des utilisateurs sont plus importants à Joulou Kriyé et Jalori (plus de $80 \%$ ) suivi du pourcentage de Issari (plus de $60 \%$ ). Cependant, ces pourcentages sont moins importants à Guelbarno et Mounoumari. Joulou kriyé et Mounimari sont des villages composés essentiellement des peulhs alors que dans le village de Issari il ya des peulhs et des Kanouris. Aucun peulh n'a été enquêté à Guelbarno et Mounoumari (Figure 2).

\section{Principaux types d'utilisations}

Types d'utilisation des rameaux de L. Pyrotechnica

Selon la population enquêtée, les rameaux de L. pyrotechnica sont plus utilisés dans le fourrage. Cependant, il existe d'autres utilisations comme la consommation, la pharmacopée et comme filtre dans les puits des villages. La consommation est plus pratiquée à Jalori, alors que la pharmacopée et le filtre sont plus pratiqués à Issari. À Guelborno, un pourcentage important de la population enquêté (40\%) ne connait pas l'utilité des rameaux de L. pyrotechnica. À Issari et Joulou Kriyé, $100 \%$ de la population enquêtée connait les types d'utilisations des rameaux de $L$. pyrotechnica. Ils sont essentiellement utilisés dans le fourrage et la consommation. Peu de personnes enquêtées méconnaissent les types d'utilisation des rameaux de $L$. pyrotechnica à Issari et Mounimari avec des pourcentages respectifs de 7,31 et $12,5 \%$. La connaissance d'utilisation des rameaux de L. pyrotechnica existe dans tous les villages enquêtés. L'utilisation dans la consommation est connue à Issari, Jalori et Joulou Kriyé. L'utilisation dans la pharmacopée est connue à Issari et Mounimari. L'utilisation dans le filtre n'est connue qu'à Issari (Tableau 1).

\section{Utilisations thérapeutiques des rameaux de} L. pyrotechnica

Toutes les trois ethnies de la zone d'étude (Haoussa, Kanouri et Peulh) utilisent les rameaux de $L$. pyrotechnica dans la thérapie traditionnelle. L'hémorroïde est traité à travers les rameaux de L. pyrotechnica par les Haoussas et les Peulhs, la jaunisse est traitée par les Kanouris et les Peulhs, les difficultés de la grossesse et de l'allaitement, les menstruations douloureuses et les démangeaisons sont traités par les peulhs, le manque d'énergie est traité par les Haoussas (Tableau 2). Cent pour cent des Kanouris enquêtés traitent la jaunisse avec les rameaux de L. pyrotechnica alors que $25 \%$ des peulh le traitent avec le même produit. 33\% des Haoussas et $25 \%$ des Peulhs enquêtés méconnaissent l'utilité thérapeutique des rameaux de L. pyrotechnica. $100 \%$ de kanouri enquêtés utilisent les rameaux de $L$. pyrotechnica dans le traitement de la jaunisse. Chez les peulhs, la plus grande utilisation thérapeutique avec les rameaux de $L$. pyrotechnica est les traitements d'hémorroïde et de la jaunisse $(25 \%$ de la population enquêtée), suivi du traitement de la menstruation douloureuse (12\%). Très peu de peulhs enquêtés $(6,2 \%)$ ont cité les traitements de la difficulté de la grossesse et de l'allaitement avec les rameaux de $L$. pyrotechnica (Tableau 2).

\section{Types d'utilisation des racines de $L$. pyrotechnica}

Beaucoup de personnes enquêtées ne connaissent pas l'utilité des racines de $L$. pyrotechnica. La grande majorité de celles qui utilisent ces racines les utilise comme bois énergie, d'autres représentées par un faible pourcentage, l'utilisent comme pharmacopée traditionnelle. À Joulou kriyé, 100\% des personnes enquêtées utilisent les racines de $L$. pyrotechnica comme bois énergie contre $65,71 \%$ à Issari, $60 \%$ à Geulbarno, 50\% à Jalori et $37,5 \%$ à Mounimari. Les racines de L. pyrotechnica sont utilisées dans la pharmacopée traditionnelle à Issari et à Mounimari avec des pourcentages respectifs de 17,14 et $25 \%$ (Figure 3 ). 


\section{Types d'utilisation d'écorce de L. pyrotechnica \\ Peu de personnes enquêtées} connaissent l'utilité de l'écorce de $L$. pyrotechnica. Cependant certains pensent qu'elle est utilisée dans la pharmacopée, le fourrage et le filtre dans les puits des villages. A Guelborno, 20\% de la population enquêtée utilisent l'écorce de L. pyrotechnica dans la pharmacopée traditionnelle. Cependant, $80 \%$ ne font aucune utilisation de l'écorce. À Issari un faible pourcentage $(8,82 \%)$ utilise l'écorce de $L$. pyrotechnica. Ces utilisations sont essentiellement liées au fourrage et à la pharmacopée traditionnelle. À Mounimari, $12,5 \%$ de la population enquêtée utilisent l'écorce de L. pyrotechnica dans le fourrage et dans la pharmacopée traditionnelle. Alors que le $75 \%$ de la population enquêtée ne fait aucune utilisation de l'écorce de cette espèce. À Jalori comme à Joulou Kriyé, aucune des personnes enquêtées n'utilise l'écorce de cette espèce (Figure 4).

\section{Utilisations thérapeutiques de l'écorce de L. pyrotechnica}

L'écorce de $L$. pyrotechnica est utilisée pour traiter l'hémorroïde par les haoussas et les peulhs, la jaunisse par les peulhs et les Kanouri et la menstruation douloureuse par les peulhs. Chez les haoussas, l'utilisation thérapeutique de l'écorce de $L$. pyrotechnica est essentiellement le traitement d'hémorroïde ( $50 \%)$. Cependant, la moitié des Haoussas enquêtés ne connait pas l'utilisation thérapeutique de l'écorce de cette espèce. Chez les peulhs, toutes les trois utilisations thérapeutiques sont représentées: hémorroïde $(23,52 \%)$, jaunisse $(23,52 \%)$ et menstruation douloureuse $(11,76 \%) .41,17 \%$ des peulhs enquêtés ne connaissent pas l'utilisation thérapeutique de l'écorce de cette espèce. $100 \%$ des kanouri enquêtés utilisent l'écorce de $L$. pyrotechnica essentiellement dans le traitement de la jaunisse (Tableau 3).

Type d'utilisation de la sève de L. pyrotechnica

Peu de personnes enquêtées connaissent l'utilité de la sève de $L$. pyrotechnica. Ainsi, ce faible pourcentage pense qu'elle est utilisée pour la consommation et dans la pharmacopée traditionnelle. À Guelborno, la sève de $L$. pyrotechnica est essentiellement utilisée dans la pharmacopée traditionnelle $(20 \%)$. À Issari, elle est utilisée dans la pharmacopée et la consommation avec des pourcentages respectifs de $15,62 \%$ et $6,25 \%$. À Mounimari, elle est aussi utilisée dans la pharmacopée et la consommation avec des pourcentages respectifs de $25 \%$ et $12,5 \%$. À Jalori et Joulou Kriyé aucune personne enquêtée ne connait l'utilité de la sève de L. pyrotechnica (Figure 5).

\section{Utilisations thérapeutiques de la Sève de L. pyrotechnica}

La majorité des thérapeutes enquêtés (67\%) ne connait pas la valeur thérapeutique de la sève de L. pyrotechnica. Cependant, certains l'utilisent pour traiter la piqure d'épine, le furoncle et l'hémorroïde. La plus grande utilisation thérapeutique de la sève de L. pyrotechnica est le traitement du Furoncle (17\% des thérapeutes enquêtés) suivi du traitement d'hémorroïde et de la piqure d'épine (8\%) des thérapeutes enquêtés (Figure 6).

\section{Type d'utilisation du bois de L. pyrotechnica}

Cent pour cent des personnes enquêtées connaissent l'utilité des bois de $L$. pyrotechnica. Le tableau des données sur l'utilisation du bois soumis à une ACP montre que les deux axes concentrent $82 \%$ de la variance totale des informations. L'axe $1 \mathrm{~s}$ 'est distingué avec $49,3 \%$ et l'axe2 avec $32,7 \%$. Les corrélations des variables avec les axes sont consignées dans le Tableau 4.

La majorité des types d'utilisation du bois est corrélée négativement avec les axes. Cependant, certains des types d'utilisation ont des corrélations positives, notamment: construction de la maison et fourrage avec l'axe 1 et bois énergie et construction de la maison avec l'axe 2. Le bois énergie est corrélé négativement avec l'axe $1(-0,39)$ et présente une corrélation positive avec l'axe 2 $(+0,30)$. La construction de la maison a des corrélations positives avec les deux axes $(+0,32$ avec l'axe 1 et $+0,063$ avec l'axe 2$)$. Le fourrage est corrélé positivement avec l'axe $1 \quad(+0,42)$ mais a une corrélation 
négative avec l'axe $2(-0,35)$. La pharmacopée a une corrélation positive avec l'axe $1(+0,35)$ et une corrélation négative avec l'axe $2(-0$ 37). La Fixation des dunes et le filtre sont corrélés négativement avec les deux axes : 0,12 et $-0,63$ respectivement pour la fixation des dunes et $-0,45$ et $-0,33$ respectivement pour le filtre.

Le bois de L. pyrotechnica est utilisé en priorité: comme bois énergie à Jalori et dans la construction des maisons à Mounimari. Cependant on l'utilise dans la fixation des dunes à Issari et dans le fourage et la pharmacopée à Guelborno. Plus le bois est utilisé dans la fixation des dunes moins il est utilisé comme bois énergie, comme fourrage et dans la pharmacopée. Plus il est utilisé dans le fourrage et la pharmacopée moins il est utilisé dans la construction de la maison et dans la fixation des dunes (Figure 7).

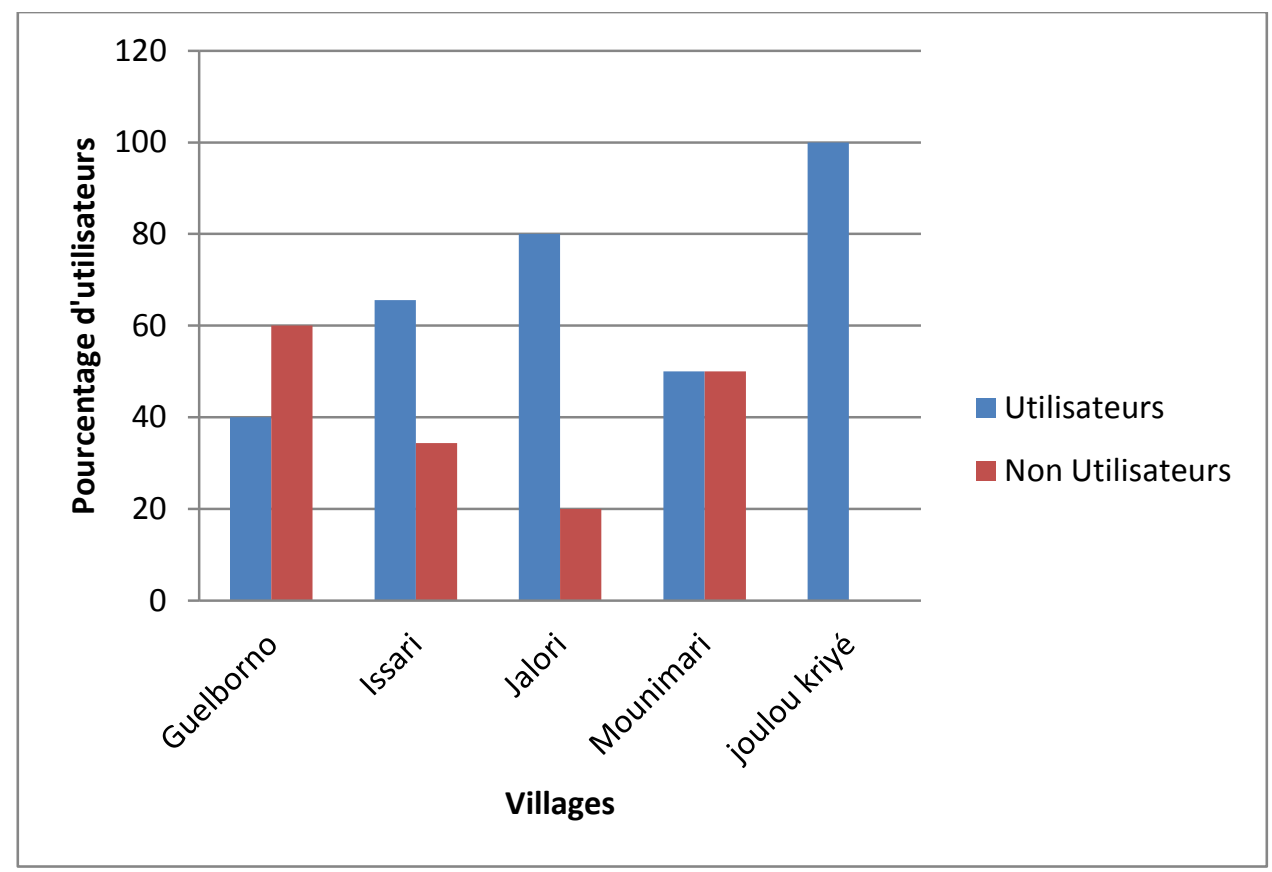

Figure 2 : Fréquence d'utilisation des organes de L. pyrotechnica.

Tableau 1 : Types d'utilisation des rameaux de L. pyrotechnica selon les villages.

\begin{tabular}{lccccc}
\hline Types d'utilisation & Guelborno & Issari & Jalori & Mounimari & Joulou Kriyé \\
\hline Fourrage & 60 & 65,8 & 66,6 & 75 & 95 \\
Assaisonnement & 0 & 4,8 & 33,3 & 0 & 5 \\
Médicinale & 0 & 19,5 & 0 & 12,5 & 0 \\
Filtre à eau & 0 & 2,4 & 0 & 0 & 0 \\
RAS & 40 & 7,3 & 0 & 12,5 & 0 \\
\hline Total & 100 & 100 & 100 & 100 & 100 \\
\hline
\end{tabular}


Tableau 2 : Utilisation des rameaux de L. pyrotechnica dans la thérapie traditionnelle.

\begin{tabular}{lccc}
\hline Symptôme et maladie & Haoussa $(\boldsymbol{\%})$ & Kanouri $(\boldsymbol{\%})$ & Peulh $(\boldsymbol{\%})$ \\
\hline Hémorroïde & 33,3 & 0 & 25 \\
Jaunisse & 0 & 100 & 25 \\
Difficulté de la grossesse et de l'allaitement & 0 & 0 & 6,2 \\
Allergie & 0 & 0 & 6,2 \\
Fortifiant & 33,3 & 0 & 0 \\
Menstruation douloureuse & 0 & 0 & 12,5 \\
RAS & 33,3 & 0 & 25 \\
\hline Total & 100 & 100 & 100 \\
\hline
\end{tabular}

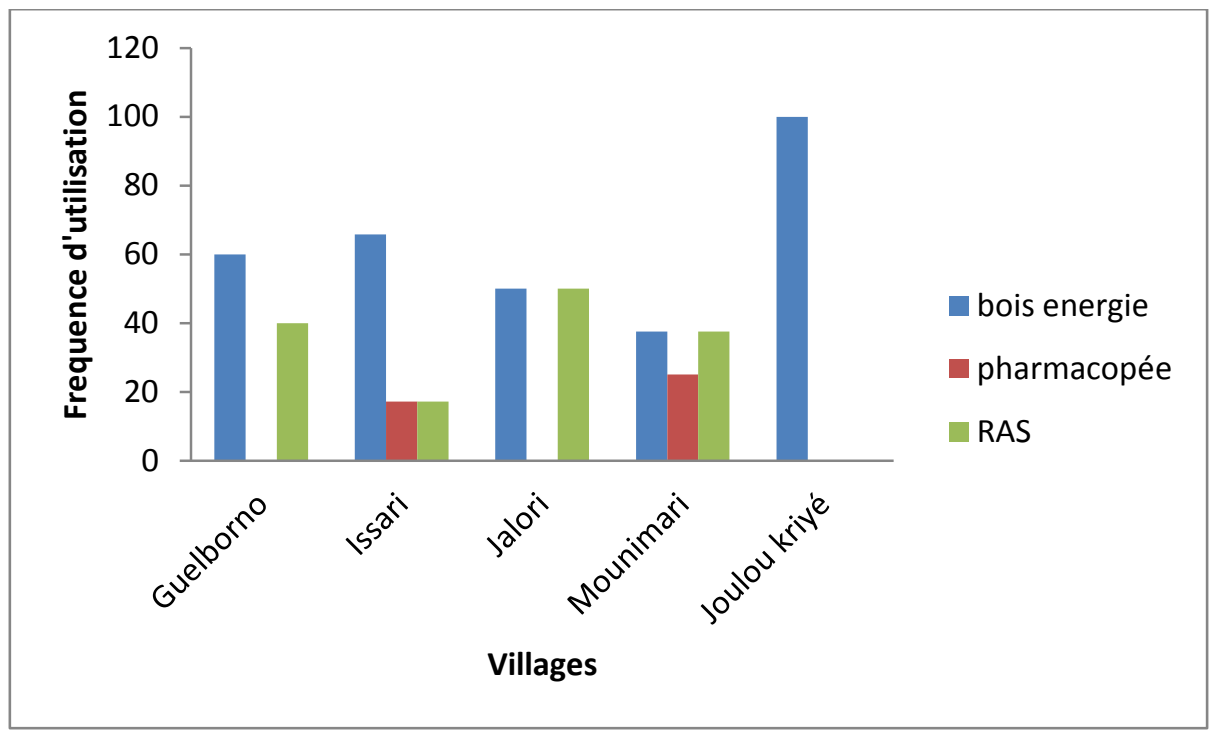

Figure 3 : Les types d'utilisations des racines de L. pyrotechnica.

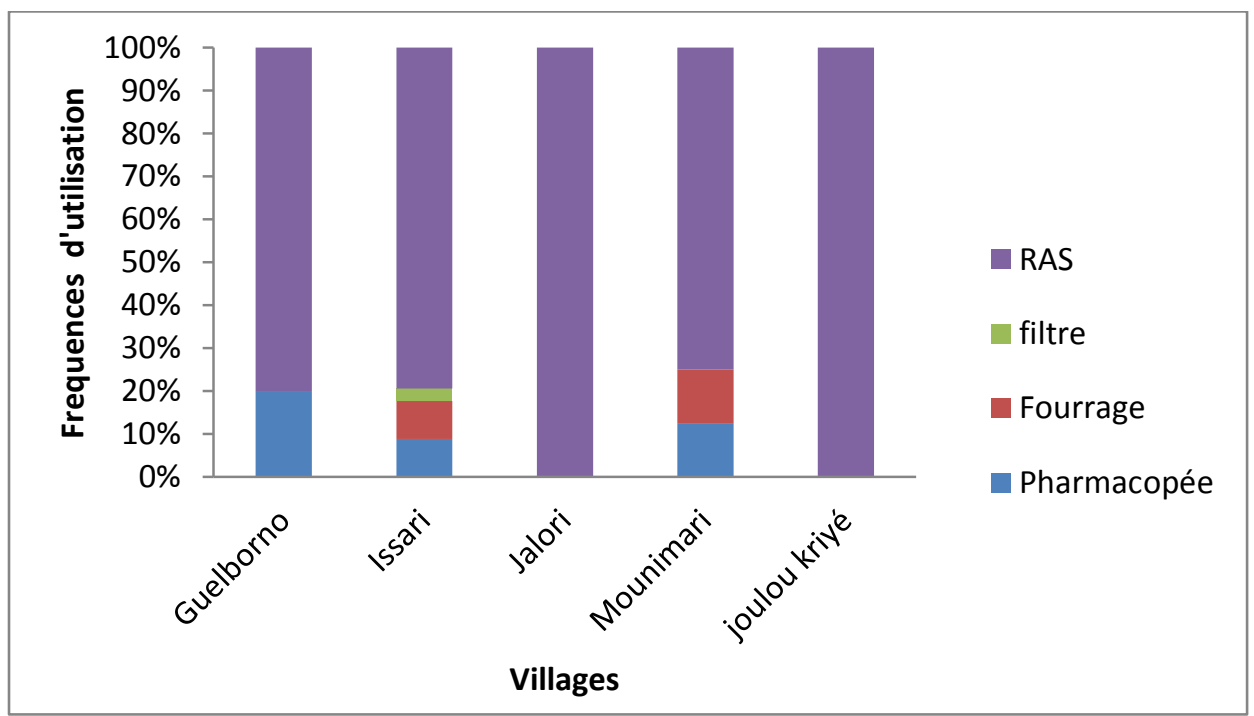

Figure 4 : Type d'utilisation de l'écorce de L. pyrotechnica. 
Tableau 3 : Utilisation thérapeutique de l'écorce de L. pyrotechnica.

\begin{tabular}{lccc}
\hline Maladies & Haoussa & Peulhs & Kanouri \\
\hline Hémorroïde & 50 & 23,52 & 0 \\
Jaunisse & 0 & 23,52 & 100 \\
Menstruation Douloureuse & 0 & 11,76 & 0 \\
RAS & 50 & 41,17 & 0 \\
\hline
\end{tabular}

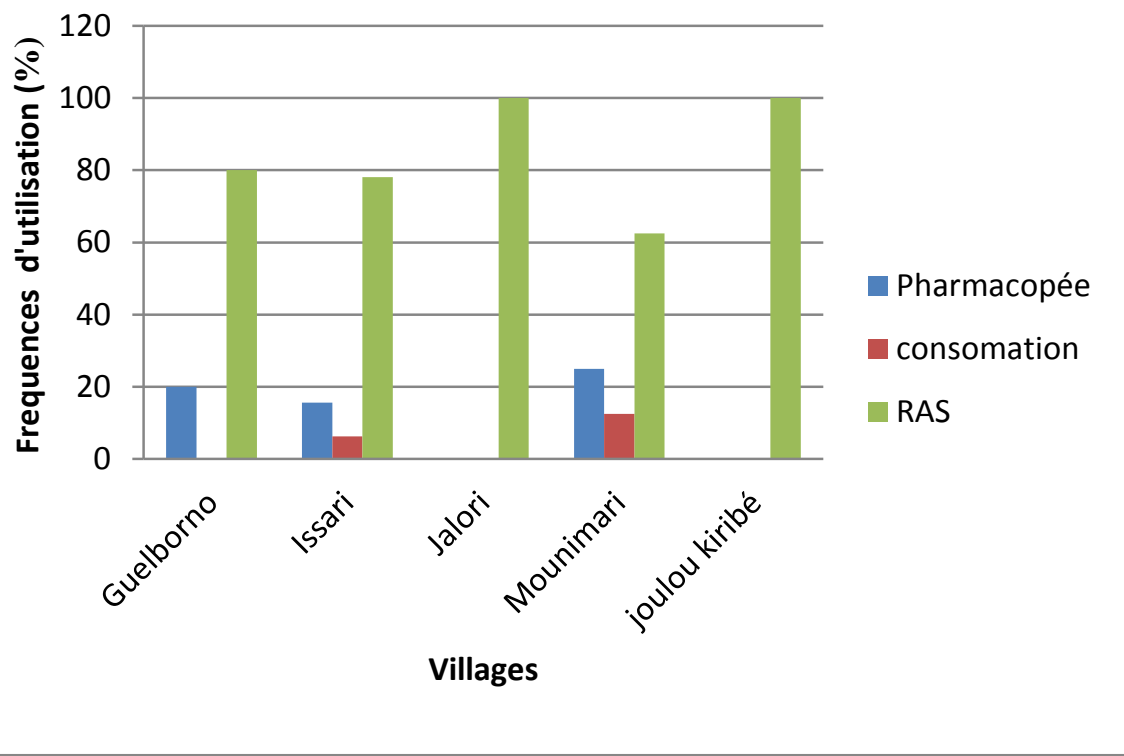

Figue 5: Types d'utilisation de la sève de L. pyrotechnica.

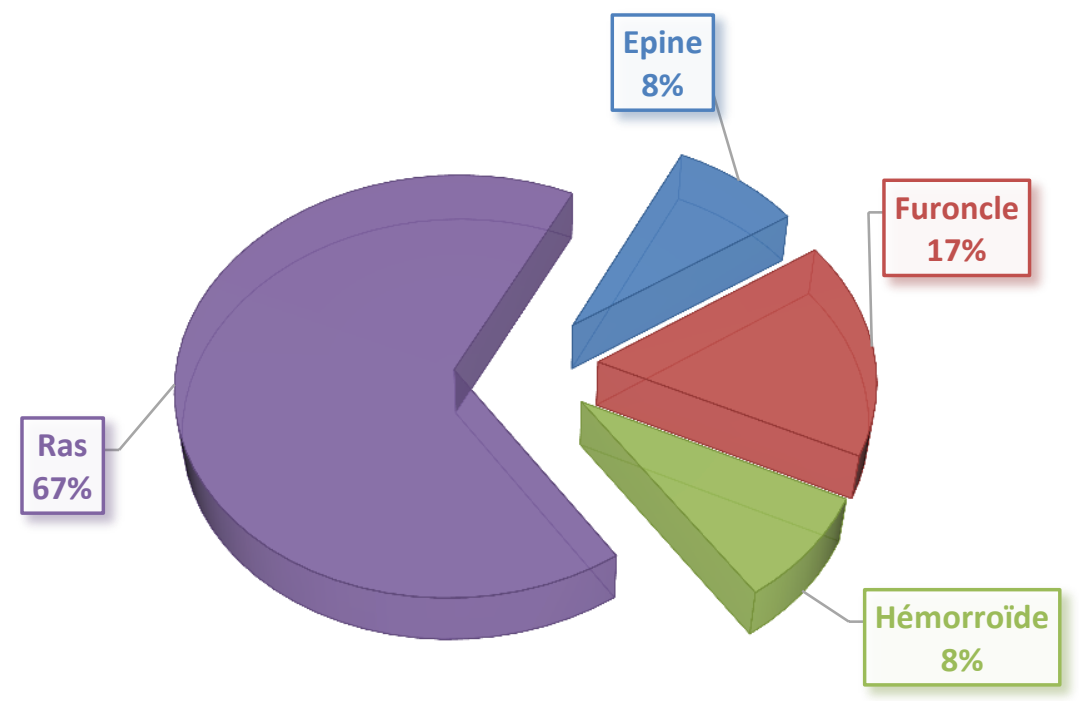

Figure 6 : Utilisations thérapeutiques de la Sève. 
Tableau 4 : Corrélation avec les axes.

\begin{tabular}{l|cc}
\hline Variable & PC1 & PC2 \\
\hline bois énergie & $-0,399^{*}$ & $0,306^{*}$ \\
construction de la & $0,324^{*}$ & 0,063 \\
maison & $0,429^{*}$ & $-0,353^{*}$ \\
Fourrage & $0,351^{*}$ & $-0,379^{*}$ \\
Pharmacopée & $-0,123$ & $-0,637^{*}$ \\
fixation des dunes & $-0,455^{*}$ & $-0,338^{*}$ \\
Filtre & $-0,455^{*}$ & $-0,338^{*}$ \\
RAS & & \\
\hline
\end{tabular}

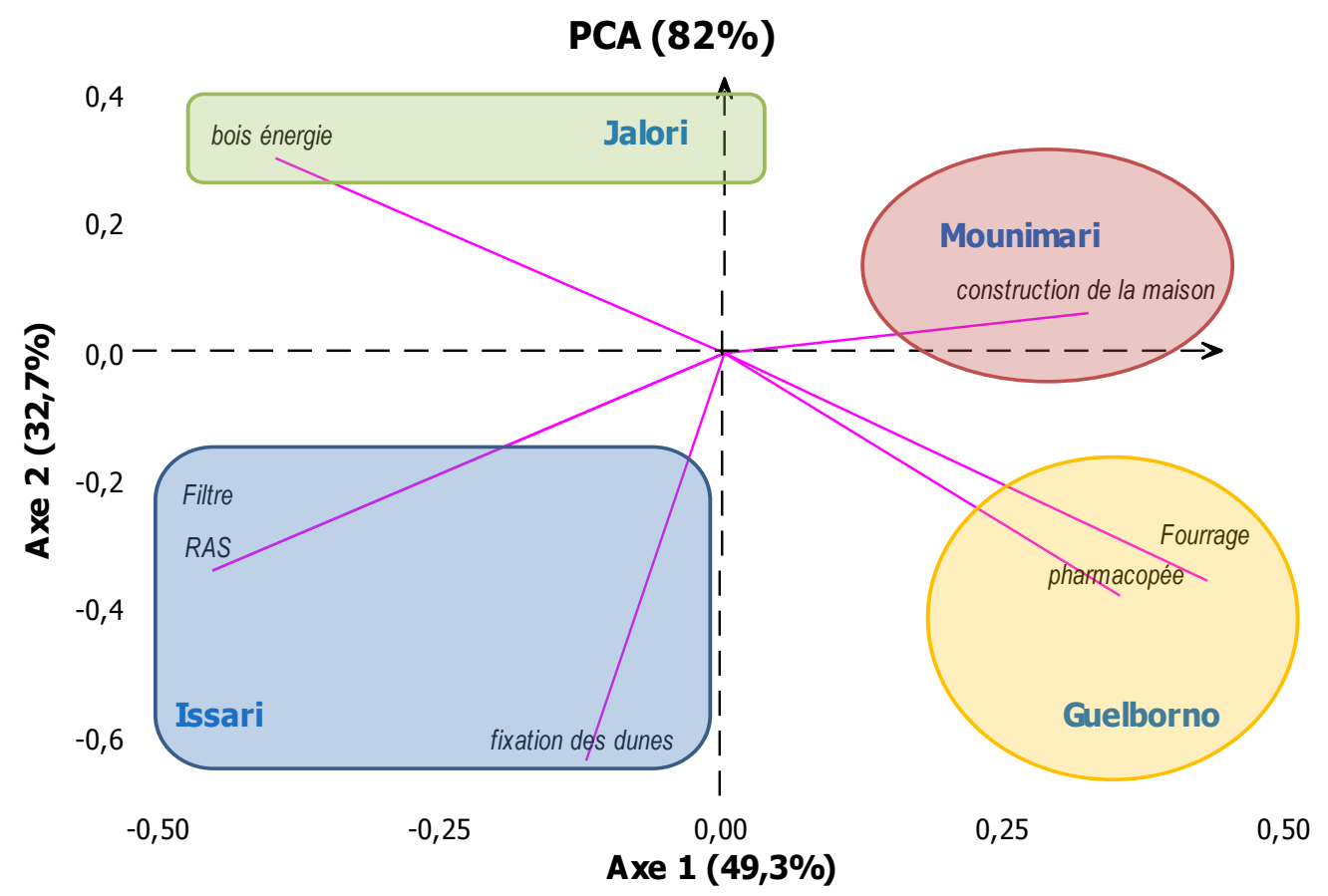

Figure 7 : ACP affectant les formes d'utilisation du bois aux localités.

\section{DISCUSSION}

La grande majorité des personnes enquêtées connait l'utilité des rameaux de $L$. pyrotechnica. Cela s'explique par le fait qu'étant dans une zone sablonneuse, $L$. pyrotechnica est l'espèce la plus représentée à cause de sa capacité d'adaptation au déficit hydrique mais aussi apte à se développer dans une zone sablonneuse. L. pyrotechnica est utilisé par la population de la zone comme fourrage. En effet, les jeunes rameaux sont consommés par les petits ruminants et les vieux rameaux par les dromadaires. Cela s'explique par le fait que l'espèce garde ses rameaux et tiges humides même pendant la période défavorable. Ce résultat est similaire au résultat de Ram (2012) sur les usages de $L$. pyrotechnica à Bikaner (Rajasthan). Selon le 
même auteur, la plante est un fourrage important; il est parcouru dans une certaine mesure par tous les herbivores, mais surtout par les chameaux. Il existe aussi d'autres utilisations comme consommation et filtre dans les puits. En effet, les jus des rameaux sont utilisés dans la préparation de la galette. Ces rameaux possèdent une grande capacité de filtrer l'eau sale pour mettre à la disposition de la population de l'eau potable. En effet les saletés sont retenues par le pouvoir absorbant de ces rameaux. Ce résultat corrobore le résultat de Ram (2016) selon qui les feuilles et les jeunes raméaux sont utilisés dans les condiments ou consommés comme légumes. L'hémorroïde est traité à travers les rameaux de L. pyrotechnica par les haoussas et les peulhs, la jaunisse est traité par les kanouris et les peulhs, les difficultés de la grossesse et de l'allaitement, les menstruations douloureuses et l'allergie sont traitées par les peulhs, le manque d'énergie est traité par les haoussas. Ce résultat complète l'étude de Gulshan et al. (2012) qui a montré l'importance des rameaux de L. pyrotechnica. Ainsi une infusion des parties aériennes est prise comme un diurétique pour traiter les troubles rénaux. Les Brindilles sont macérées et le liquide est pris pour traiter la rétention urinaire. Le jus des feuilles est traditionnellement utilisé pour le traitement de l'asthme, les rhumatismes et la diarrhée infantile. Pour enlever l'épine, la pâte de feuille est préparée et appliquée sur la blessure d'épine. Ce résultat confirme le résultat de Ram (2016) selon qui les parties aériennes sont prises comme diurétique pour traiter les troubles rénaux, les calculs rénaux et la toux. Les ramilles sont macérées et le liquide est bu pour Traiter la rétention urinaire.

Dans notre étude la plus part des personnes enquêtées ne connait pas l'utilité des racines de L. pyrotechnica. Cela peut être dû au fait que la disponibilité de la partie aérienne de la plante fait en sorte que les gens ne s'intéressent pas aux racines. Cependant dans le village de Mounimari, le pourcentage des utilisateurs de la racine est plus élevé. La plus part des utilisateurs les utilise comme bois d'énergie. Cependant certains les utilisent dans la pharmacopée traditionnelle. Ainsi elles sont utilisées pour traiter l'hémorroïde, les démangeaisons et les maladies du au génie par les peulhs; l'hémorroïde et la jaunisse par les peulhs et les haoussas et la gonoquoxie par les haoussas. Ce résultat complète l'étude de Schmeizer et Gurib-Fakim (2013) qui a montré l'utilité médicinale des racines de $L$. pyrotechnica, qui selon lui, sont utilisées pour le traitement de l'asthme, la constipation et les maux d'estomac. Elles sont également utilisées pour traiter la stérilité, pour prévenir l'avortement spontané et comme diurétique pour traiter les maladies vénériennes. Selon Ram (2016), la décoction de racine est prise pour traiter la constipation et les coliques. Elles sont utilisées pour traiter les maux d'estomac, pour prévenir spontanément l'avortement, pour traiter la stérilité et comme diurétique pour traiter les maladies vénériennes. Selon Thomas (2012), la racine de Leptadenia hastata, espèce du même genre que L. pyrotechnica, est utilisée pour traiter la gale. Selon toujours le même auteur, Leptadenia hastata est utilisée pour le traitement des maladies de la peau, l'hypertension et l'impuissance.

L'utilité de l'écorce de $L$. pyrotechnica n'est pas très bien connue par la population enquêtée. Cela est peut-être dû au fait que la tige de l'espèce étant toujours humide, la récolte de l'écorce devient compliquer. Cependant certains l'utilisent dans la pharmacopée, le fourrage et le filtre dans les puits des villages. En ce qui concerne l'utilisation médicinale, l'écorce de $L$. pyrotechnica est utilisée pour traiter l'hémorroïde par les haoussas et les peulhs, la jaunisse par les peulhs et les Kanouri et la menstruation douloureuse par les peulhs. $\mathrm{Ce}$ résultat complète le résultat de Verma et al. (2014) qui ont montré l'utilité médicinale de l'écorce de cette espèce. Ainsi au Pakistan, une décoction de tige est prise comme antihistaminique et un expectorant tandis qu'en Inde, elle est utilisée pour traiter la goutte et les rhumatismes. Plusieurs auteurs dont Gulshan et al. (2012) et Schmeizer et Gurib-Fakim (2013) ont montré l'utilité médicinale de l'écorce de L. pyrotechnica. L'écorce de racine mélangée avec du lait de 
vache est utilisée comme purgatif. Selon l'étude réalisée par Khasawneh et al. (2011) sur antioxydant, anti-lipoxygénase et activité cytotoxique de L. pyrotechnica (Forssk.), les feuilles et l'écorce de la plante sont utilisées dans la médecine traditionnelle au Mali pour préparer des remèdes antispasmodiques, antiinflammatoires et antibactériens. Dans notre étude, la plus part des personnes enquêtées méconnait l'utilité de la sève de $L$. pyrotechnica. Cependant certains l'utilisent pour la consommation et dans la pharmacopée traditionnelle. La majorité des thérapeutes enquêtés ne connait pas la valeur thérapeutique de la sève de L. pyrotechnica. Cela peut être dû au fait que l'espèce est plus utilisée comme bois énergie ou fourrage. Néanmoins les peulhs l'utilisent pour traiter la piqure d'épine, le furoncle et l'hémorroïde. Ce qui confirme l'idée de Gulshan et al. (2012) qui ont montré que la sève de $L$. pyrotechnica est appliquée sur la peau pour traiter la variole, le psoriasis, l'eczéma et la dermatite. Selon l'idée de Ram (2016), la sève de $L$. pyrotechnica est frottée sur la peau pour traiter la variole et la dermatite. Le bois de $L$. pyrotechnica est utilisé en priorité comme bois énergie à Jalori et dans la construction des maisons à Mounimari. Cependant on l'utilise dans la fixation des dunes à Issari et dans le fourrage et la pharmacopée à Guelborno. Bois de bonne qualité pour la cuisine, le bois de L. pyrotechnica peut prendre feu même étant humide. Le bois de L. pyrotechnica est aussi apprécié pour la cuisine car il est facile à couper et à transporter. Sa flexibilité fait qu'il est apprécié pour la construction de la maison par le peulhs. A l'état humide, le bois de L. pyrotechnica est très apprécié par les grands ruminants comme les dromadaires. La forte capacité de régénération de l'espèce fait que le bois est choisie pour la fixation des dunes dans les zones ensablées.

\section{Conclusion}

Cette étude sur l'importance ethnobotanique de L. pyrotechnica a montré que toutes les parties de L. pyrotechnica sont utilisées. Ce qui montre la grande pression qui pèse sur l'espèce. Cependant certaines utilisations sont plus destructrices que d'autres. Parmi les utilisations faites de $L$. pyrotechnica, les plus destructrices sont la coupe pour la fixation des dunes qui diminue voir empêche la résilience des formations à $L$. pyrotechnica. Si la Technique appropriée pour la coupe n'est pas respectée, cela conduira à la destruction irréversible voir la disparition de l'écosystème et La transhumance, à travers la consommation des grands ruminants comme les dromadaires et les piétinements des régénérations de l'espèce par les animaux. Les autres utilisations sont aussi destructrices mais la pression n'est pas assez forte pour entraver la résilience de l'écosystème. L'étude suggère à ce qu'une norme de prélèvement de $L$. pyrotechnica soit définie pour la fixation des dunes et que la capacité de charge des formations à $L$. pyrotechnica soit contrôlée vis-à-vis des transhumances pour une conservation durable de l'espèce.

\section{CONFLIT D'INTERETS}

Les auteurs déclarent qu'il n'y a aucun conflit d'intérêts entre eux.

\section{CONTRIBUTIONS DES AUTEURS}

AAESI a assuré la collecte et les traitements des données, l'analyse et la rédaction du manuscrit. HR et IS ont apporté des conseils méthodologiques à la réalisation du travail et ont révisé le manuscrit. MKAH a contribué dans la préparation du premier DRAFT et la révision du manuscrit. AM a coordonné toutes les activités de cette recherche et a contribué dans la révision de la version finale de l'article.

\section{REFERENCES}

Abdou HMK, Rabiou H, Abdou L, Abdourahamane IS, Sanoussi IAZ, Soumana A., Mahamane A. 2019. Germination et croissance des plantules d'une espèce fruitière indigène au Niger: Balanites aegyptiaca(L.) Del. Int. J. Biol. Chem. Sci., 13(2) : 693-703. DOI: https://dx.doi.org/10.4314/ijbcs.v13i2.10

Abdou L, Dan Guimbo I, Larwanou M, Inoussa MM, Mahamane A. 2014. Utilisation de Prosopis africana (G. et Perr.) Taub. Dans le sud du département d'Aguié au Niger: les différentes formes et leur importance. Int. J. Biol. Chem. 
Sci, 3(8): 1065-1074. DOI: http://dx.doi.org/10.4314/ijbcs.v8i3.20

Assogbadjo AE, Glèlè KR, François HA, Akomian FA, Gbèlidji FV, Tina KJT, Codjia C. 2011. Ethnic differences in use value and use patterns of the threatened multipurpose scrambling shrub (Caesalpinia bonduc L.) in Benin. Journal of Medicinal Plants Research, 5(9): 549-1557. DOI: http://www.academicjournals.org/JMPR

Bellefontaine R, Petit S, Pain-Orcet M, Deleporte P, Bertault JG. 2001. Les arbres hors forêt. Vers une meilleure prise en compte. Cahier FAO Conservation, 35: 231p.

Boubacar MM. 2010. Variabilité spatiale de la productivité des parcours pastoraux dans le Département de Gouré. Mémoire de Diplôme d'Etudes Approfondies (DEA), Université Abdou Moumouni de Niamey, 81 pages.

Fahim U, Muhammad U, Bachir AC, Zafar UZ. 2015. Phytochemical and Pharmacological Studies of Leptadenia pyrotechnica. Pakistan Journal of Pharmaceutical Research, 2(1): 78-85. DOI: http://pjpr.net

FSRGTT. 2010. Rapport Générale du Forum sous Régionale sur la transhumance transfrontalière Tenu à Gogounou du 14 au 16 Avril 2010, 101 pages. DOI : www.inter-reseaux.org

Glèlè RK, Assogbadjo AE, Sinsin B, Pelz D. 2009. Structure spatiale et régénération naturelle de Pterocarpus Erinaceus Poir. en zone Soudanienne au Benin. Revue Ivoirienne des Sciences et Technologies, 13: 199-212. DOI : http://www.revist.ci

Gulshan K, Thommandru B, Moye-Rowley WS. 2012. Proteolitic degradation of the yap transcription factor is regulated by subcellular localization and the E3 ubiquitin ligase Not4. J. Biol. Chem, 287(32): 26796-26805. DOI: https://www.ncbi.nlm.nih.gov

Khasawneh MA, Elwy HM, Hamza AA, Fawzi NM, Hassan AH. 2011. Antioxidant, antilipoxygenase and cytotoxic activity of Leptadenia pyrotechnica (Forsk.) Decne polyphenolic constituents. Molecules, (16): 7510-7521. DOI: 10.3390/molécules 16097510

Niaz S, Bokhari TZ, Sherwani SK, Younis U, Dasti AA. 2013. Ethnobotanical study of some medicinal plants of thal desert Punjab. Pakistan Int J. Pharm. Res. Bioscience, 2(6): 31-41. DOI: www.ijprbs.com

PADL-Diffa, 2010. Projet de développement local de la région de Diffa Un bel exemple du partenariat BAD/Niger, Unité des relations extérieurs et de la communicatio/yal 36 pages

Planetoscope, 2019. Statistique mondiale en temps réels. https://outilstice.com/2019/02.

Rabiou H, Bationo BA, Kossi A, Adzo DK, Mahamane A, Kouami K. 2017. Perception paysanne et importance socioculturelle et ethnobotanique de Pterocarpus erinaceus au Burkina Faso et au Niger. Afrique Science, 13(5): 4360. DOI: http://www.afriquescience.info

Ram B. 2016. Important uses of Leptadenia pyrotechnica of Bikaner. International Journal of Advances in Science Engineering and Technology, 4(4): 2628. DOI: http://iraj.in

Schmelzer GH, Gurib-Fakim A. 2013. Ressources végétales de l'Afrique tropicale, plantes medicinale 2 (11) 418 pp.

Thomas SD. 2012. Leptadenia hastata: A Review of its Traditionnel uses and its Pharmacological Activity Medicinal chemistry. Open Access Journal, 2(7): 148-150. DOI: $\quad 10.4172 / 2161-$ 0444.1000132

Verma N, Jha KK, Chaudhary S, Singh O, Kumar A. 2014. Phytochemistry, pharmacology and traditional uses of Leptadenia pyrotechnica an important medicinal plant. Indian J. Pharm. Bio. Res., (2): 128-134. DOI : 10.30750/ijpbr.20.1.20. 